OPEN ACCESS

Edited by:

Massimo Broggini,

Mario Negri Pharmacological

Research Institute (IRCCS), Italy

Reviewed by:

Phei Er Saw,

Sun Yat-sen Memorial Hospital, China Junhua Mai,

Houston Methodist Research Institute, United States

*Correspondence: Juliana Hamzah juliana.hamzah@perkins.org.au

Specialty section:

This article was submitted to Cancer Molecular Targets and Therapeutics,

a section of the journa

Frontiers in Oncology

Received: 16 December 2021 Accepted: 20 January 2022 Published: 22 February 2022

Citation:

Yeow YL, Wu J, Wang $X$, Winteringham L, Feindel KW,

Tirnitz-Parker JEE, Leedman PJ, Ganss $R$ and Hamzah J (2022) ECM Depletion Is Required to Improve the Intratumoral Uptake of Iron Oxide Nanoparticles in Poorly Perfused Hepatocellular Carcinoma. Front. Oncol. 12:837234. doi: 10.3389/fonc.2022.837234

\section{ECM Depletion Is Required to Improve the Intratumoral Uptake of Iron Oxide Nanoparticles in Poorly Perfused Hepatocellular Carcinoma}

\author{
Yen Ling Yeow ${ }^{1}$, Jiansha $W^{1}{ }^{1}$, Xiao Wang ${ }^{1}$, Louise Winteringham ${ }^{1}$, Kirk W. Feindel ${ }^{2}$, \\ Janina E. E. Tirnitz-Parker ${ }^{3}$, Peter J. Leedman ${ }^{1}$, Ruth Ganss ${ }^{1}$ and Juliana Hamzah ${ }^{1,3^{*}}$

\footnotetext{
1 Harry Perkins Institute of Medical Research, Centre for Medical Research, QEll Medical Centre, The University of Western Australia, Nedlands, WA, Australia, ${ }^{2}$ Centre for Microscopy, Characterisation and Analysis, The University of Western Australia, Nedlands, WA, Australia, ${ }^{3}$ Curtin Medical School and Curtin Health Innovation Research Institute, Curtin University, Bentley, WA, Australia
}

Improving tumor access for drug delivery is challenging, particularly in poorly perfused tumors. The availability of functional tumor blood vessels for systemic access is vital to allow drugs or imaging agents to accumulate in the tumor parenchyma. We subjected mice engineered to develop hepatocellular carcinoma (HCC), to treatment with tumor necrosis factor alpha (TNF $\alpha$ ) conjugated to a CSG peptide (CSGRRSSKC). CSG binds to the laminin-nidogen-1 complex of the extracellular matrix (ECM) in HCC. When produced as a recombinant fusion protein, the TNF $\alpha$-CSG functions as an ECM depletion agent via an immune-mediated mechanism to improve tumor perfusion. Tumor perfusion in HCC was dramatically improved after daily intravenous (i.v.) injection of $5 \mu \mathrm{g}$ TNF $\alpha$-CSG for five consecutive days. Following treatment, we assessed the tumor accessibility to accumulate an imaging agent, superparamagnetic iron-oxide nanoparticles (IO-NP). Here, we compared the passive delivery of an i.v. dose of IO-NP in HCC following ECM depletion after TNF $\alpha$-CSG treatment, to the intratumoral accumulation of a comparable dose of CSG-targeted IO-NP in HCC with intact ECM. Magnetic resonance imaging (MRI) $\mathrm{T}_{2}$-weighted scans and $\mathrm{T}_{2}$ relaxation times indicate that when the tumor $\mathrm{ECM}$ is intact, HCC was resistant to the intratumoral uptake of IO-NP, even when the particles were tagged with CSG peptide. In contrast, pre-treatment with TNF $\alpha$-CSG resulted in the highest IO-NP accumulation in tumors. These findings suggest poorly perfused HCC may be resistant to molecular-targeted imaging agents including CSG-IO-NP. We demonstrate that specific ECM depletion using TNF $\alpha$-CSG improves nanoparticle delivery into poorly perfused tumors such as HCC.

Keywords: extracellular matrix, peptide targeting, hepatocellular carcinoma, nanoparticles, tumor necrosis factor, immune cells, perfusion, magnetic resonance imaging 


\section{INTRODUCTION}

Systemic delivery of therapeutics and imaging agents in tumors relies primarily on accessibility via tumor blood vessels (1). The passive delivery of non-targeted agents in tumors is based on the enhanced permeability and retention (EPR) effect of leaky tumor vasculature, resulting in higher accumulation in tumors than in normal tissues (2). However, this passive delivery via the EPR effect is insufficient to allow macromolecules and nanoparticles to extravasate and accumulate in the parenchyma of solid tumors (3). More recently, ligands with specific binding affinity to unique molecular tumor targets have been developed for the delivery of therapeutic and imaging payloads including nanoparticle-based contrast agents (4). We have previously reported a tumor extracellular matrix (ECM)-targeting peptide, CSG, that specifically binds to the aberrant network of lamininnidogen-1 complex in a number of mouse and human solid tumors including breast, pancreatic and liver cancers (5). When CSG is linked to superparamagnetic iron-oxide nanoparticles (IO-NP), CSG-IO-NP accumulate selectively in the stromal $\mathrm{ECM}$, for instance in pancreatic neuroendocrine tumors (PNET) (6).

However, intratumoral binding to specific molecular targets relies on a functional circulation via tumor blood vessels. This remains a challenge for clinical and experimental tumors that are heterogeneously perfused and thus represents a significant barrier to drug delivery (7-11). In particular, tumors that develop in the liver, including hepatocellular carcinoma (HCC) are difficult to target for nanoparticle delivery $(8-13)$. Whilst the normal liver is very well-perfused through hepatic arterial and portal venous blood flows, HCC nodules are fed primarily via arterial blood supply $(8,13-15)$. Hence, intravenously injected nanoparticles accumulate preferentially in the normal liver (10, 13). Furthermore, the heterogeneity of blood perfusion in HCC can impede intratumoral uptake of nanoparticles. Dysplastic nodules and early-stage HCC are hyper-vascularized and wellperfused but late-stage and poorly differentiated HCC showed significant decrease in arterial blood supply. Injection of imaging materials detectable by contrast-enhanced computed tomography, ultrasonography and magnetic resonance imaging (MRI) revealed a perfusion defect in advanced-staged HCC compared to the non-cancerous regions $(8,10,11)$. Therefore, the use of nanoparticle-based imaging contrast enhancement in HCC diagnosis is often based on non-specific accumulation in the hepatic tissues and small peritumoral vessels surrounding the tumors $(16,17)$.

We have previously shown that CSG-IO-NP targets the stromal ECM in a PNET tumor model and is a contrast agent superior to untargeted IO-NP in detecting tumor stroma by MRI (6). In addition, we have developed a therapeutic approach to improve tumor perfusion in PNET and breast cancer models using an immune modulating cytokine tagged to CSG peptide, TNF $\alpha$-CSG. TNF $\alpha$-CSG treatment induces immune-mediated ECM depletion, which alleviates the compression on the tumor blood vessels and enhances tumor perfusion. TNFo-CSG-treated tumors are then more accessible for systemic uptake of nanoparticles (5).
In this study, we assessed the accumulation of CSG-IO-NP and untargeted IO-NP in poorly perfused HCC with intact ECM, and the passive uptake of IO-NP following ECM-depletion by TNF $\alpha$-CSG treatment. Our findings indicate that IO-NP have poor access to HCC, even when the particles were targeted with CSG peptide. However, IO-NP accumulation in HCC is significantly improved following pre-treatment with TNF $\alpha$ CSG. Thus, we demonstrate that TNF $\alpha$-CSG is a useful ECMdepletion agent that can significantly improve HCC perfusion and facilitate the passive delivery of IO-NP.

\section{METHODS}

\section{Animal Models}

ALB-Tag mice (on a $\mathrm{C} 3 \mathrm{H}$ background) express the oncogene SV40 Large $\mathrm{T}$ antigen (Tag) under the control of the mouse albumin gene promoter (ALB), and develop spontaneous HCC as previously described (18). RIP1-Tag5 mice express Tag under the control of the rat insulin gene promoter (RIP) in pancreatic $\beta$ cells, and develop spontaneous PNET as previously described (19). ALB-Tag or RIP1-Tag5 mice with advanced tumor nodules were used at $\approx 10-12$ or $\approx 29-30$ weeks of age, respectively. All mice were kept under pathogen-free and temperature-controlled conditions in individually ventilated cages, exposed to a 12-hour day-to-night cycle. A minimum of $\mathrm{n}=3$ mice per group were used in each study. All animal experiments were performed in accordance with the Australian code for the care and use of animals for scientific purposes at the University of Western Australia (UWA) Animal Facility with approval from the UWA Animal Ethics Committee.

\section{In Vivo Lectin Perfusion}

Mice were intravenously (i.v., tail vein) injected with $1 \mu \mathrm{g} / \mu \mathrm{L}$ of tomato lectin (Lycopersicon esculentum conjugated to either FITC or DyLight ${ }^{\circledR}$ 594; Vector). After 10 min of circulation, the mice were heart-perfused with $2 \%$ neutral-buffered formalin (w/v) and tumors with surrounding normal tissue were frozen in the O.C.T. compound (Tissue-Tek ${ }^{\circledR}$ ).

\section{CSG Peptide and TNF $\alpha$-CSG Synthesis}

Synthetic carboxyfluorescein (FAM)-labelled CSG (molecular weight $=1,612.17$ ) was kindly provided by Dr. Vankata Ramana Kotamraju (Sanford Burnham Prebys Medical Discovery Institute, La Jolla, CA, USA). Recombinant murine TNF $\alpha$-CSG $(18.9 \mathrm{kDa})$ was produced as described previously $(5,20)$.

\section{In Vivo Peptide and TNF $\alpha-C S G$ Binding}

Tumor-bearing mice were injected i.v. with $100 \mu \mathrm{L}$ of either $1 \mathrm{mM}$ FAM-CSG, FITC-TNF $\alpha$-CSG or FITC-TNF $\alpha$ in phosphate buffered saline (PBS). After $1 \mathrm{~h}$, animals were euthanised and subjected to transcardial perfusion with sterile PBS to remove unbound peptides or proteins. Tissues, including tumor, heart, liver, spleen, kidney, vertebrae, lung and pancreas were excised and imaged under a UV illuminator (Illumatool, Lightools Research, CA, USA) for assessment of green fluorescence intensity. 
Tissues were embedded in O.C.T. (Tissue-Tek ${ }^{\circledR}$ ) and stored at $-80^{\circ}$ $\mathrm{C}$ for histology analysis.

\section{TNF $\alpha-C S G$ Treatment}

Tumor-bearing mice were i.v. injected once per day with PBS, CSG $(0.8 \mu \mathrm{g})$, or TNF $\alpha$-CSG $(5 \mu \mathrm{g})$ for 4 to 5 consecutive doses. Tumors were analyzed for immune cell infiltration, lectin perfusion and IO-NP uptake within $48 \mathrm{~h}$ after final treatment, unless stated otherwise.

\section{Analysis of Intratumoral Nanoparticle Uptake by Magnetic Resonance Imaging}

Superparamagnetic iron oxide nanoparticles (IO-NP) with and without CSG conjugation were prepared and analyzed as previously published $(6,21-23)$. Untargeted IO-NP (6) were injected i.v. $(100 \mu \mathrm{L}, 5 \mathrm{mg} / \mathrm{kg} \mathrm{Fe})$ in tumor-bearing mice within $24 \mathrm{~h}$ after final treatment with TNF $\alpha$-CSG or CSG peptide. In parallel, untreated tumor-bearing mice received an i.v. dose of CSG-IO-NP as described previously (6). After $4 \mathrm{~h}$ circulation, mice were perfused with sterile PBS. Tissues were collected, fixed in $2 \%$ formalin for $2 \mathrm{~h}$ and embedded in $2 \%$ agarose. Ex vivo MRI scans were performed at 9.4 $\mathrm{T}$ with a Bruker BioSpec 94/30 magnet, Avance III HD console and ParaVision 6.0.1 acquisition software as described previously $(5,6) . \mathrm{T}^{*}$ and $\mathrm{T} 2$ parameter maps were calculated from the MGE and MSME datasets, respectively, using the ParaVision 6.0.1 macro fitinlsa, which fits the signal for each pixel according to a mono-exponential decay. Image analysis was performed using ImageJ. Tumor volume and IO-NP-induced changes in MRI signal level were measured by using the Image Display and Processing Tool. Regions of interest (ROI) for each tumor was manually defined using the track tool, based on the MSME image with TE $=8 \mathrm{~ms}$. Statistical analyses for tumor volumes were obtained by combining the ROI statistics on an image slice by slice.

\section{Flow Cytometry Analysis}

Tumors and normal liver tissues were excised from PBS-control and TNF $\alpha$-CSG-treated C3H and ALB-Tag mice, minced and incubated in Dulbecco's Modified Eagle Medium (DMEM) highglucose medium containing $0.1 \mathrm{mg} / \mathrm{ml} \mathrm{DNase} \mathrm{I} \mathrm{(Sigma)} \mathrm{and} 0.5$ $\mathrm{mg} / \mathrm{ml}$ collagenase IV (Sigma) for $1 \mathrm{~h}$ at $37^{\circ} \mathrm{C}$ under gentle rotation. The cell suspension was passed through a $70-\mu \mathrm{m}$ membrane filter and subsequently washed with FACS buffer [1\% BSA (w/v, Sigma) in PBS]. For analysis of immune cells, viable cells were stained with the following antibodies: CD11bA488 (M1/70, BD Pharmingen, 1:100), CD68-A647 (FA-11, Biolegend, 1:100), CD8-FITC (53-6.7, eBioscience, 1:100), CD4-FITC (GK1.5, eBioscience, 1:100) and CD45-A700 (30F11, eBioscience, 1:100). Cells were imaged on a BD SORP Fortessa and analyzed on BD FACSDiva software version 8.0.1 (BD Biosciences, USA).

\section{Histology Analysis}

Tissue distribution of bound lectin, FAM-CSG and FITC-TNF $\alpha$ CSG was detected on $8 \mu \mathrm{m}$ tissue sections based on their fluorescence intensity. The intensity of FITC-TNF $\alpha$-CSG and FITC-TNF $\alpha$ in tissues were amplified with anti-fluorescein antibody (polyclonal, LifeTechnologies). For co-staining analysis, the following antibodies were used: anti-SV40 Large T antigen antibody (polyclonal, Santa Cruz, 1:100) anti-CD31 (390; ebioscience, 1:100), anti-laminin (polyclonal; Millipore, 1:200) and anti-nidogen-1 (polyclonal; Millipore, 1:100), CD11b (M1/ 70, BD Pharmingen), CD68 (FA-11, Abcam), CD8 (53-6.7, eBioscience, 1:100) and CD4 (GK1.5, eBioscience, 1:100). For secondary detection, fluorescence-labelled 488-, 594- or 647conjugated anti-rat, rabbit or goat IgG (LifeTechnologies) were used. Images were captured on a Nikon Ti-E microscope (Nikon Instrument Inc., NY, USA) or 3DHISTECH Slide Scanner (3DHISTECH, Budapest, Hungary). Image analysis and quantification were performed using NIS software modules (version 4.0).

\section{Statistical Analysis}

Statistical analyses were performed using GraphPad Prism 7 (GraphPad Prism Software, San Diego, CA, USA). Data were analyzed by the Student's t-test (two-tailed) or one-way analysis of variance (ANOVA) as indicated. A p value $<0.05$ was considered statistically significant. Error bars indicate standard error of the mean (SEM). Experiments were carried out in an unblinded fashion.

\section{RESULTS}

\section{Accessibility of ALB-Tag HCC to Lectin, CSG Peptide and TNF $\alpha-C S G$}

Intratumoral perfusion requires functional blood vessels. We first evaluated blood vessel functionality in ALB-Tag HCC in comparison with PNET in RIP1-Tag5 mice using i.v.-injected lectin binding to blood vessels as a surrogate marker for tumor perfusion. Figure 1A compares the ALB-Tag and RIP1-Tag5 tumors that were positive for SV40 T antigen. Whilst all vessels in the surrounding normal liver were positive for lectin, ALB-Tag HCC showed only weak traces of lectin-painted blood vessels. In contrast, RIP1-Tag5 tumors visibly displayed lectin-painted blood vessels. This finding suggests that the ALB-Tag HCC is more difficult to access systemically than RIP1-Tag5 tumors.

However, as shown in Figure 1B, i.v.-injected fluoresceinconjugated CSG peptide (FAM-CSG), homed specifically into HCC but not other normal tissues including liver. In HCC, FAM-CSG colocalized with its target receptor, the laminin and nidogen-1 complex, which is expressed in tumor ECM (Figure 1C). As indicated by nidogen-1 staining in Figure 1D, HCC expressed CSG receptor at levels 25-fold higher than the normal liver. In addition, the i.v.-injected FITC-labeled TNF $\alpha$ CSG also accumulated strongly in HCC compared to the liver tissue (Figure 1E). The unconjugated TNF $\alpha$ showed limited tumor uptake (Figure 1E). These data demonstrate that CSGtagged molecules accumulate in HCC tumor parenchyma, and when conjugated to TNF $\alpha$, have the potential to influence the ECM perfusion barrier. 


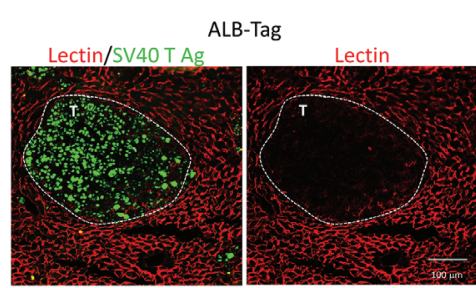

B
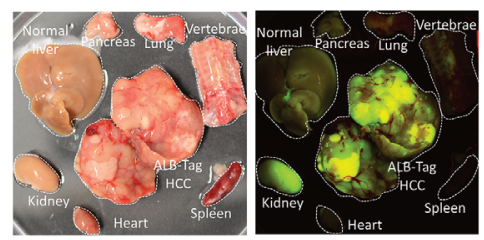

C

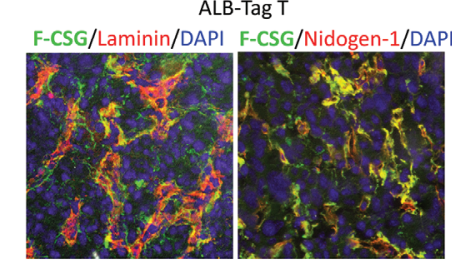

RIP1-Tag5

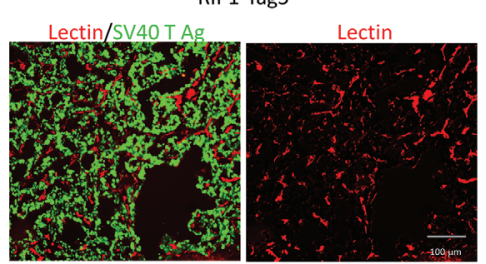

Normal liver

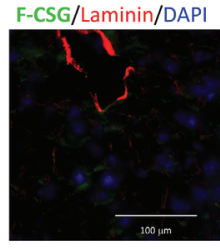

D

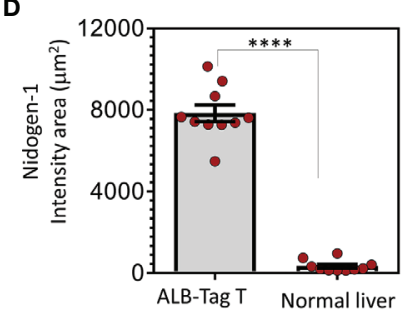

$\mathbf{E}$

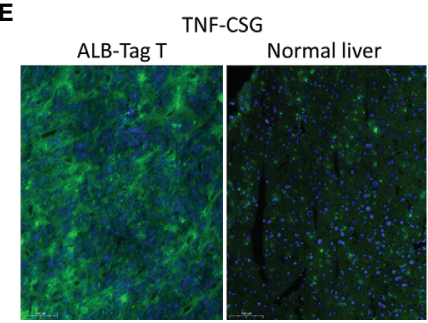

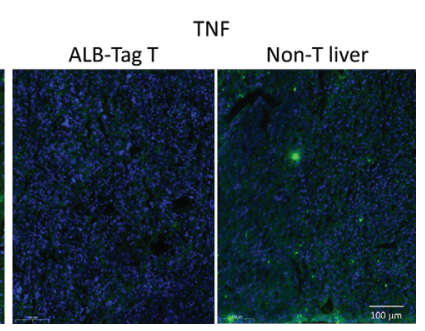

FIGURE 1 | HCC accessibility to lectin and binding specificity to CSG peptide and TNF $\alpha$-CSG. (A) Lycopersicon Esculentum (Tomato) Lectin DyLight ${ }^{\circledR} 594$ was injected i.v. in mice bearing ALB-Tag HCC or RIP1-Tag5 tumors. Representative micrographs on indicated tissues show lectin-painted vessels (red) in tumors (T) stained with anti-SV4O Large T antigen antibody (green). (B) Mice bearing ALB-Tag HCC were i.v. injected with 0.1 mmol of FAM-CSG, and tissues were collected after $1 \mathrm{~h}$ of circulation. Photographic image of collected tissues under bright light (left) and UV illuminator (right) are shown. Peptide binding is shown in tumors (green) but not in normal tissues. The kidney is the clearance organ. (C) HCC tumors from ALB-Tag mice and normal liver from C3H mice were co-stained for laminin and nidogen-1. Representative micrographs show FAM-CSG binding (green) and laminin or nidogen-1 expression (red). Co-localisation is indicated in yellow. Scale bar $100 \mu \mathrm{m}$. (D) Quantitative analysis of nidogen-1 staining per field of each tumor or normal liver, as shown in panel (C) and mean \pm SEM ( ${ }^{\star \star \star * P}<0.0001$ by Student's t-test). (E) Analysis of FITC-TNF $\alpha$-CSG and FITC-TNF $\alpha$ binding in vivo detected with anti-FITC antibody (green) and nuclear staining (DAPI) in indicated tissues. Scale bars: $25 \mu \mathrm{m}$.

\section{Immune-Mediated Effects of TNF $\alpha-C S G$ in Improving HCC Perfusion}

We have previously demonstrated that directing TNF $\alpha$-CSG to tumor ECM triggers immune cell infiltration which in turn delivers a cocktail of ECM-degrading proteases leading to specific tumor ECM breakdown (5). To assess the intratumoral effect of TNF $\alpha$-CSG in HCC, ALB-Tag mice and age-matched wild-type $\mathrm{C} 3 \mathrm{H}$ mice were treated with an i.v. dose of $5 \mu \mathrm{g}$ TNF $\alpha$ CSG per day for five consecutive days. Tumors from ALB-Tag mice and normal liver from $\mathrm{C} 3 \mathrm{H}$ mice were analyzed two days after the final treatment for immune infiltrates, ECM content and lectin binding (Figure 2). ALB-Tag HCC tumors treated with TNF $\alpha$-CSG showed significantly higher infiltration of macrophages $\left(\mathrm{CD} 68^{+} / \mathrm{CD} 11 \mathrm{~b}^{+}\right), \mathrm{CD}^{+}$and $\mathrm{CD}^{+} \mathrm{T}$ cells compared to PBS-treated tumors (gating strategy and FACS plots are shown in Supplementary Figure S1). The TNF $\alpha$ CSG-induced immune cell infiltration was specific to tumors as the immune cell status in normal liver tissue remained unaffected by the TNF $\alpha$-CSG treatment (Figures 2A, B). Intratumorally, the immune infiltrates accumulated mainly in ECM areas positive for nidogen-1 expression (Figure 2B).

Next, we assessed changes to the tumor ECM content in response to TNF $\alpha$-CSG treatment. Data in Figure 2C highlight that nidogen-1 staining in TNF $\alpha$-CSG-treated tumors was significantly lower than in the control PBS-treated tumors. This ECM-depletion effect is consistent with our previous data in other tumor models (5). To determine the effect of ECM reduction on tumor perfusion, lectin binding to tumor blood vessels was compared in TNF $\alpha$-CSG- treated versus control tumors. Figure 2D shows representative tumors with lectinpainted blood vessels co-stained for the blood vessel marker CD31. The indicated micrographs show a lack of lectin-staining in HCC with intact ECM, even though the tumors were positive for vessel staining (CD31). TNF $\alpha$-CSG-treated tumors show visible lectin-painted blood vessels $\left(\operatorname{lectin}^{+} / \mathrm{CD} 31^{+}\right)$. The quantitative analysis showed a 6 -fold increase in lectin-painted tumor blood vessels in TNF $\alpha$-CSG-treated HCC (Figure 2D). These data demonstrate that TNF $\alpha$-CSG improves blood vessel function in HCC. 


\section{Accessibility of IO-NP in Intact and ECM- Depleted HCC}

Next, we assessed the intratumoral uptake of CSG-IO-NP in HCC by performing ex vivo MRI scans of the ALB-Tag HCC tumors following $4 \mathrm{~h}$ in vivo circulation of CSG-IO-NP. In parallel, we include the MRI scans of ALB-Tag HCC injected with untargeted IO-NPs, following TNF $\alpha$-CSG or CSG-peptide control treatment at doses and dosing frequencies as described in Figure 2. Figure 3A illustrates $\mathrm{T}_{2}{ }^{*}$ and $\mathrm{T} 2$ relaxation images of scanned HCC based on their small (diameter $<5 \mathrm{~mm}$ ) and large (diameter $>5 \mathrm{~mm}$ ) sizes. Based on changes in the $\mathrm{T} 2$ relaxation time which indicate the relative amount of IO-NP in tumors, our data (Figure 3B) demonstrate that CSG-IO-NP did not effectively accumulate in HCC, unlike previously reported in the RIP1-Tag5 tumors (6). There was no significant difference between CSG-IO-NP and the untargeted IO-NP in HCC with intact ECM. However, our data showed that TNF $\alpha-C S G$ pretreatment induced significantly higher IO-NP accumulation in HCC, when compared to control tumors treated with either CSG-targeted or untargeted IO-NP.

We further confirmed the distribution of IO-NP in HCC by immunofluorescence analysis. Figure 4 shows micrographs of HCC with and without ECM depletion, immunologically stained for traces of FAM-labelled CSG-IO-NP and FAM-IO-NP and distribution of infiltrating macrophages $\left(\mathrm{CD} 68^{+}\right)$. The micrographs depict higher traces of IO-NP in TN $\alpha$-CSGtreated HCC compared to CSG-IO-NP in the HCC with intact ECM (Figure 4A). The IO-NP distribution in response to TNF $\alpha$ -

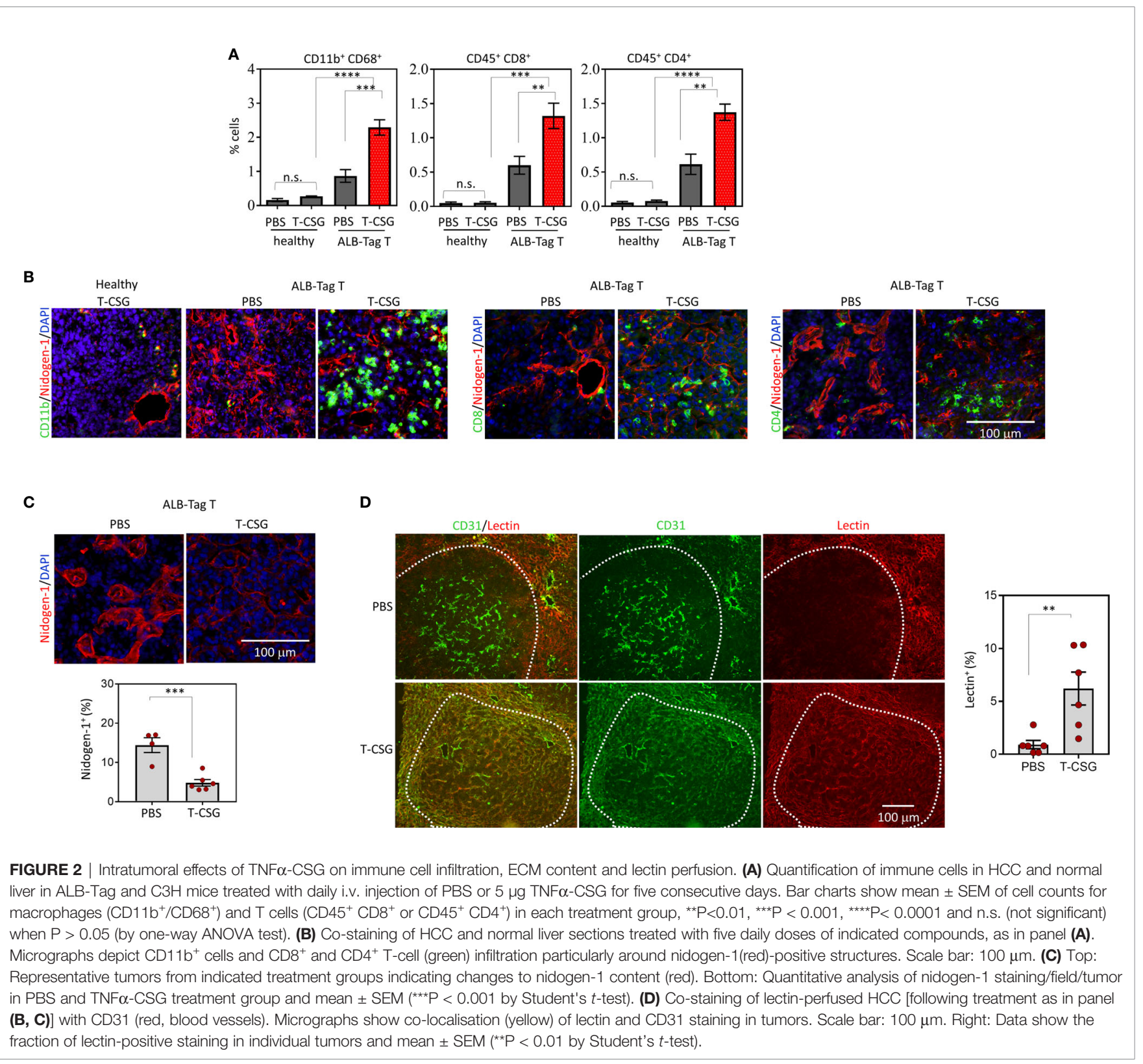


A

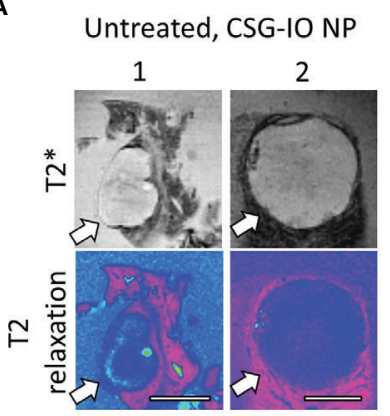

CSG-treated, IO NP

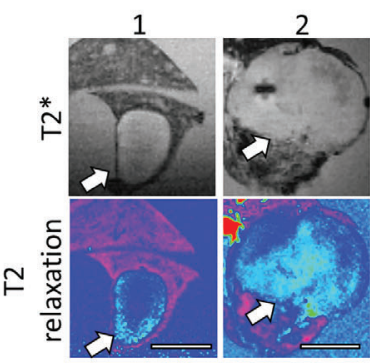

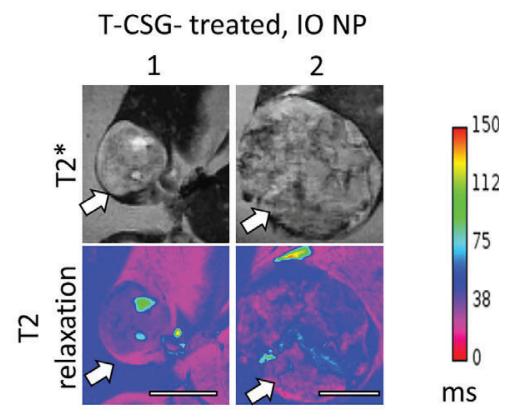

B

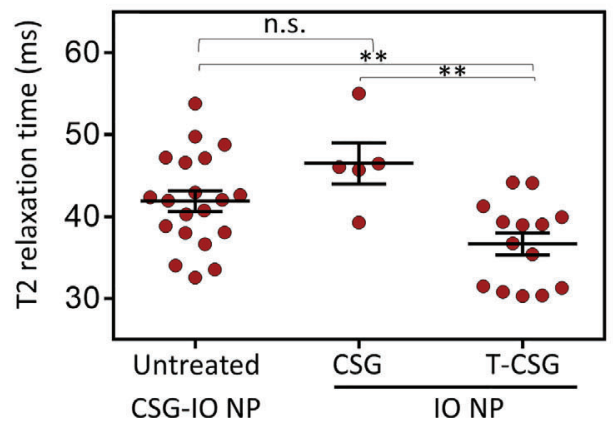

FIGURE 3 | Accumulation of CSG-IO-NP and IO-NP in HCC with intact and/or reduced ECM detected by MRI. (A) Representative images of T2*-weighted (top) and T2 relaxation maps (magenta, bottom) from ex vivo MRI scans of liver with HCC (tumor sizes, $<5 \mathrm{~mm}$ and $>5 \mathrm{~mm}$ in diameter, indicated by arrow). Scale bars: 4 $\mathrm{mm}$. Left: HCC with intact ECM after $4 \mathrm{~h}$ in vivo circulation of CSG-IO-NP. Middle: HCC treated with $5 \times 0.8 \mu \mathrm{g}$ CSG peptide and then i.v.-injected with untargeted IO-NP. Right: HCC treated with $5 \times 5 \mu \mathrm{g}$ TNF $\alpha$-CSG and then i.v.-injected with untargeted IO-NP. (B) Reduction in T2 relaxation time indicates the relative increase of IO-NP in individual tumors and mean \pm SEM per group (**P $<0.01$ and $n . s$. when $\mathrm{P}>0.05$ by one-way ANOVA test).

CSG treatment exceeds the number of $\mathrm{CD}^{+} 8^{+}$macrophages (Figure 4B), suggesting that intratumoral accumulation of IONP was independent of phagocytic uptake of the nanoparticles by the infiltrating macrophages. Our findings suggests that pretreatment with perfusion-promoting agents, such as TNF $\alpha$-CSG, may improve the delivery of nanoparticles especially for poorly perfused tumors, such as ALB-Tag HCC.

\section{DISCUSSION}

Our study demonstrates that whilst EPR may be sufficient for some small drug molecules to penetrate solid tumors, poorly perfused tumors may remain inaccessible for larger molecules, including nanoparticles such as IO-NP. Even when IO-NP were tagged with CSG peptide, which is known to improve intratumoral uptake through CSG-specific binding to the aberrantly expressed tumor ECM (6), poorly perfused ALBTag HCC remained resistant to IO-NP uptake. IO-NP particles mainly accumulated in the normal liver. However, a short treatment with TNF $\alpha$-CSG to increase tumor perfusion resulted in significant improvement in the accumulation of IONP in the otherwise impenetrable HCC. Therefore, in addition to the normal clearance activity of the liver, these findings highlight that inadequate perfusion of the tumor represents a major barrier that may significantly affect drug delivery and treatment efficacy in HCC.

Systemically injected agents, including nanoparticles, circulate in the blood until they are cleared from the circulation and eliminated from the body either by renal clearance, or by the liver via hepatobiliary elimination (15). The liver clearance pathway makes it particularly challenging to develop strategies for the delivery of agents for liver-associated diseases such as HCC. Hence, the drug delivery approach for HCC needs to take into consideration both passive and active mechanisms to improve intratumoral delivery and to minimize non-specific uptake and rapid elimination by the liver (14). Here, we found that HCC tissues are accessible to small molecules including CSG $(<1.5 \mathrm{kDa})$ and TNF $\alpha$-CSG $(18.9 \mathrm{kDa})$ but less accessible to lectin conjugates $(>70 \mathrm{kDa})$ and larger molecules such as IO-NP.

Our data indicate that CSG, as a free peptide, effectively escapes the rapid and non-specific liver uptake. CSG is an excellent HCC-targeting agent as it binds specifically to aberrant tumor ECM and not to the normal ECM. However, the use of CSG as HCC-targeting agent for intratumoral delivery is greatly influenced by the type and size of payloads. HCC 
A
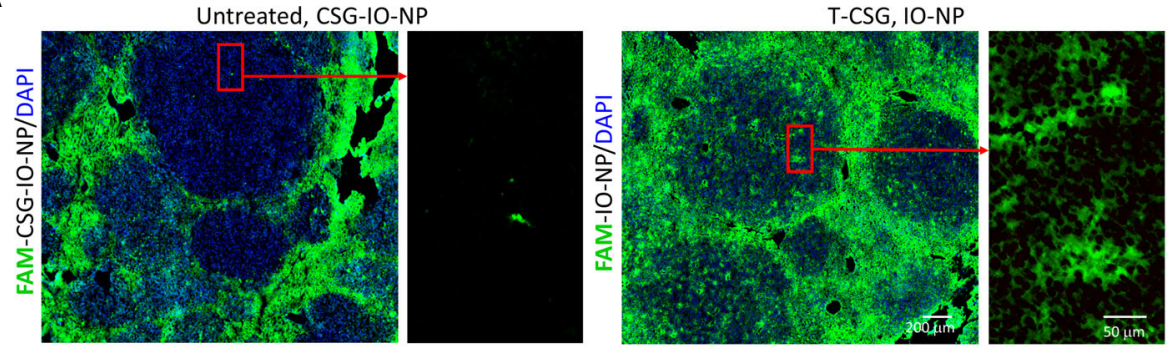

B

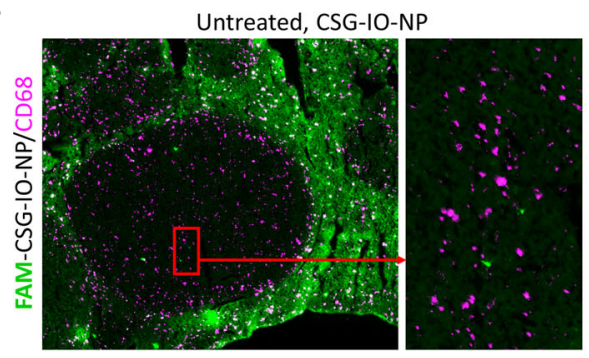

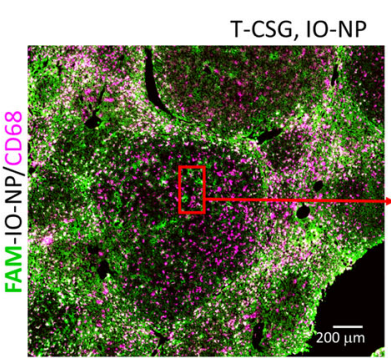

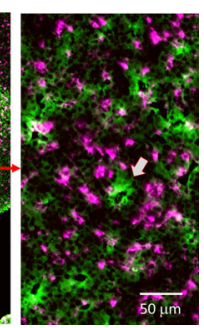

FIGURE 4 | Intratumoral localisation of IO-NP. Liver tissue sections from indicated treatment group $4 \mathrm{~h}$ after an i.v. injection of FAM-labelled CSG-IO-NP and IO-NP (as described in Figure 3) were analyzed for IO-NP distribution. (A) Representative micrographs show detection of FAM-labelled CSG-IO-NP and IO-NP, which were amplified by immunostaining with anti-FITC antibody (green). Rectangular box: Selected regions in HCC were compared for IO-NP accumulation at higher magnification. (B) Corresponding tissues in (A) were co-stained for CD68 ${ }^{+}$cells (infiltrating macrophages, red). Arrow: An area within tumor positive for IO-NP that lacks CD68 ${ }^{+}$cells. Scale bars: 200 and $50 \mu \mathrm{m}$.

tissues are inaccessible to the CSG targeted IO-NP, an agent that we have previously shown to be highly effective for intratumoral delivery in tumors with high ECM content such as PNET (6). Similar to the untargeted IO-NP, the CSG-IO-NP mostly accumulated in the non-malignant liver. This finding suggests that the rate of hepatic nanoparticle uptake is quicker and more efficient than CSG-IO-NP access and accumulation in tumors. Our data are consistent with previous reports that found nonspecific IO-NP accumulation to be a major challenge for nanomedicines, irrespective of the physical and chemical properties of nanoparticles such as careful control over their surface coating to improve blood half-life, and incorporation of active targeting moieties with specific ligands for enhanced tumor binding (12).

Furthermore, like other treatment-resistant cancers, HCC is comprised of dense stroma with excessive production of ECM $(24,25)$. Since HCC develops in the setting of cirrhosis in the vast majority of cases, the excessive fibrotic changes with significant matrix deposition and disruption of the hepatic architecture make it extremely challenging for therapeutic agents to penetrate and reach the target cancer cells $(26,27)$. Our ALB-Tag tumors display some of these microenvironmental features, including the high ECM content and poor perfusion, despite the high level of vascularization of ALB-Tag HCC. TNF $\alpha$-CSG treatment of mice bearing ALB-Tag HCC increased intratumoral immune cell infiltrates, reduced ECM content and improved tumor perfusion.
The observed intratumoral effects in response to TNF $\alpha$-CSG are consistent with our previous findings in other solid tumor models (5). The ECM-depletion in these tumors was triggered by the protease secretion through increased immune cell infiltration (5). Similar protease-mediated ECM breakdown is likely to occur in HCC, as the TNF $\alpha$-CSG-treated ALB-Tag tumors showed reduced ECM content. Our ECM-depletion approach to improve tumor perfusion using TNF $\alpha$-CSG is also consistent with other ECM-reducing strategies to enhance perfusion and increase access to solid tumors (28-31).

An important application of TNF $\alpha$-CSG treatment may be to sensitize HCC for improved systemic access of nanoparticles such as IO-NP and potentially other HCC-specific therapeutics. CSG binding is conserved in human HCC (5), and thus in addition to its use to enhance diagnostic imaging, TNF $\alpha$-CSG has the therapeutic potential to improve patient outcome. Currently, most patients are diagnosed with HCC at an advanced stage, when systemic therapy is the only treatment option. The commonly used systemic therapies are the tyrosine kinase inhibitors, sorafenib and lenvatinib, both of which have significant associated toxicity and provide only a marginal survival benefit $(32,33)$. We have shown that CSG targeting of TNF $\alpha$ dramatically rescued the systemic toxicity associated with untargeted TNF $\alpha$ (5), and therefore ECM-depletion by TNF $\alpha$ CSG is viable and safe in a preclinical setting. Our data suggest that TNF $\alpha$-CSG may provide a much-needed novel approach to 
improve treatment efficacy by increasing systemic access to these drugs.

\section{DATA AVAILABILITY STATEMENT}

The original contributions presented in the study are included in the article/Supplementary Material. Further inquiries can be directed to the corresponding author.

\section{ETHICS STATEMENT}

The animal study was reviewed and approved by the University of Western Australia Animal Ethics Committee.

\section{AUTHOR CONTRIBUTIONS}

Conceptualization, JH and RG. Investigation, YY, JW, XW, and KF. Funding acquisition, JH, RG, and PL. Supervision, JH, KF, and LW. Writing, JH. Review and editing, JH, RG, LW, PL, and JT-P. All authors have read and agreed to the published version of the manuscript.

\section{FUNDING}

This research was supported by Cancer Council WA project grant, Perkins Ride to Conquer Cancer, Liver Cancer Collaboration, NHMRC

\section{REFERENCES}

1. Choi IK, Strauss R, Richter M, Yun CO, Lieber A. Strategies to Increase Drug Penetration in Solid Tumors. Front Oncol (2013) 3:193. doi: 10.3389/ fonc.2013.00193

2. Matsumura Y, Maeda H. A New Concept for Macromolecular Therapeutics in Cancer Chemotherapy: Mechanism of Tumoritropic Accumulation of Proteins and the Antitumor Agent Smancs. Cancer Res (1986) 46:6387-92.

3. Prabhakar U, Maeda H, Jain RK, Sevick-Muraca EM, Zamboni W, Farokhzad OC, et al. Challenges and Key Considerations of the Enhanced Permeability and Retention Effect for Nanomedicine Drug Delivery in Oncology. Cancer Res (2013) 73:2412-7. doi: 10.1158/0008-5472.CAN-12-4561

4. Ruoslahti E. Peptides as Targeting Elements and Tissue Penetration Devices for Nanoparticles. Adv Mater (2012) 24:3747-56. doi: 10.1002/adma. 201200454

5. Yeow YL, Kotamraju VR, Wang X, Chopra M, Azme N, Wu J, et al. ImmuneMediated ECM Depletion Improves Tumour Perfusion and Payload Delivery. EMBO Mol Med (2019) 11:e10923. doi: 10.15252/emmm.201910923

6. Chopra M, Wu J, Yeow YL, Winterinham L, Clemons TD, Saunders M, et al. Hamzah, Enhanced Detection of Desmoplasia by Targeted Delivery of Iron Oxide Nanoparticles to the Tumour-Specific Extracellular Matrix. Pharmaceutics (2021) 13(10):1663. doi: 10.3390/pharmaceutics 13101663

7. Gillies RJ, Schornack PA, Secomb TW, Raghunand N. Causes and Effects of Heterogeneous Perfusion in Tumors. Neoplasia (1999) 1:197-207. doi: 10.1038/sj.neo.7900037

8. Asayama Y, Yoshimitsu K, Nishihara Y, Irie H, Aishima S, Taketomi A, et al. Arterial Blood Supply of Hepatocellular Carcinoma and Histologic Grading: Radiologic-Pathologic Correlation. AJR Am J Roentgenol (2008) 190:W28-34. doi: 10.2214/AJR.07.2117

9. Hectors SJ, Wagner M, Bane O, Besa C, Lewis S, Remark R, et al. Quantification of Hepatocellular Carcinoma Heterogeneity With Multiparametric Magnetic Resonance Imaging. Sci Rep (2017) 7:2452. doi: 10.1038/s41598-017-02706-Z project grants (APP1058073, APP1121131, APP1157240) to JH and RG, and a Cancer Research Institute Clinic and Laboratory Integration Program (CLIP) Grant to RG. JH and RG are the recipients of the Cancer Council Western Australia Research Fellowship and a Woodside Energy Cancer Research Fellowship, respectively.

\section{ACKNOWLEDGMENTS}

We thank Meenu Chopra for performing the MRI imaging studies and Ji Li for assisting with FACS analysis. The authors acknowledge the facilities, and the scientific and technical assistance of Microscopy Australia at the Centre for Microscopy, Characterisation and Analysis, The University of Western Australia, a facility funded by the University, State and Commonwealth Governments.

\section{SUPPLEMENTARY MATERIAL}

The Supplementary Material for this article can be found online at: https://www.frontiersin.org/articles/10.3389/fonc.2022.837234/ full\#supplementary-material

Supplementary Figure 1 | Gating strategies and flow cytometry plots of quantification of $\mathrm{CD} 11 \mathrm{~b}^{+} / \mathrm{CD} 8^{+}, \mathrm{CD}^{+} / \mathrm{CD} 45^{+}$and $\mathrm{CD} 4^{+} / \mathrm{CD} 45^{+}$in normal liver (from $\mathrm{C} 3 \mathrm{H}$ mice) and ALB-Tag HCC tumors following i.v. injections of PBS or TNF $\alpha$ CSG ( $5 \mu \mathrm{g}$ dose/day) for 5 consecutive days.

10. Imai $Y$, Murakami T, Yoshida S, Nishikawa M, Ohsawa M, Tokunaga K, et al. Superparamagnetic Iron Oxide-Enhanced Magnetic Resonance Images of Hepatocellular Carcinoma: Correlation With Histological Grading. Hepatology (2000) 32:205-12. doi: 10.1053/jhep.2000.9113

11. Yang D, Li R, Zhang XH, Tang CL, Ma KS, Guo DY, et al. Perfusion Characteristics of Hepatocellular Carcinoma at Contrast-Enhanced Ultrasound: Influence of the Cellular Differentiation, the Tumor Size and the Underlying Hepatic Condition. Sci Rep (2018) 8:4713. doi: 10.1038/ s41598-018-23007-z

12. Haute DV, Berlin JM. Challenges in Realizing Selectivity for Nanoparticle Biodistribution and Clearance: Lessons From Gold Nanoparticles. Ther Deliv (2017) 8:763-74. doi: 10.4155/tde-2017-0057

13. Tanimoto A, Kuribayashi S. Application of Superparamagnetic Iron Oxide to Imaging of Hepatocellular Carcinoma. Eur J Radiol (2006) 58:200-16. doi: 10.1016/j.ejrad.2005.11.040

14. Baboci L, Capolla S, Di Cintio F, Colombo F, Mauro P, Dal Bo M, et al. The Dual Role of the Liver in Nanomedicine as an Actor in the Elimination of Nanostructures or a Therapeutic Target. J Oncol (2020) 2020:4638192. doi: $10.1155 / 2020 / 4638192$

15. Poon W, Zhang YN, Ouyang B, Kingston BR, Wu JLY, Wilhelm S, et al. Elimination Pathways of Nanoparticles. ACS Nano (2019) 13:5785-98. doi: 10.1021/acsnano.9b01383

16. Andreou C, Neuschmelting V, Tschaharganeh DF, Huang CH, Oseledchyk A, Iacono P, et al. Imaging of Liver Tumors Using Surface-Enhanced Raman Scattering Nanoparticles. ACS Nano (2016) 10:5015-26. doi: 10.1021/ acsnano.5b07200

17. Unterrainer M, Eze C, Ilhan H, Marschner S, Roengvoraphoj O, SchmidtHegemann NS, et al. Recent Advances of PET Imaging in Clinical Radiation Oncology. Radiat Oncol (2020) 15:88. doi: 10.1186/s13014-020-01519-1

18. Ryschich E, Lizdenis P, Ittrich C, Benner A, Stahl S, Hamann A, et al. Molecular Fingerprinting and Autocrine Growth Regulation of Endothelial Cells in a Murine Model of Hepatocellular Carcinoma. Cancer Res (2006) 66:198-211. doi: 10.1158/0008-5472.CAN-05-1636 
19. Ganss R, Hanahan D. Tumor Microenvironment can Restrict the Effectiveness of Activated Antitumor Lymphocytes. Cancer Res (1998) 58:4673-81.

20. Johansson A, Hamzah J, Payne CJ, Ganss R. Tumor-Targeted TNFalpha Stabilizes Tumor Vessels and Enhances Active Immunotherapy. Proc Natl Acad Sci USA (2012) 109:7841-6. doi: 10.1073/pnas.1118296109

21. Kang YS, Risbud S, Rabolt JF, Stroeve P. Synthesis and Characterization of Nanometer-Size Fe3O4 and $\gamma$-Fe2O3 Particles. Chem Mater (1996) 8:2209-11. doi: $10.1021 / \mathrm{cm} 960157 \mathrm{j}$

22. Starmans LW, Burdinski D, Haex NP, Moonen RP, Strijkers GJ, Nicolay K, et al. Iron Oxide Nanoparticle-Micelles (ION-Micelles) for Sensitive (Molecular) Magnetic Particle Imaging and Magnetic Resonance Imaging. PloS One (2013) 8:e57335. doi: 10.1371/journal.pone.0057335

23. Yu M, Huang S, Yu KJ, Clyne AM. Dextran and Polymer Polyethylene Glycol (PEG) Coating Reduce Both 5 and $30 \mathrm{Nm}$ Iron Oxide Nanoparticle Cytotoxicity in 2D and 3D Cell Culture. Int J Mol Sci (2012) 13:5554-70. doi: 10.3390/ijms13055554

24. Henke E, Nandigama R, Ergun S. Extracellular Matrix in the Tumor Microenvironment and Its Impact on Cancer Therapy. Front Mol Biosci (2019) 6:160. doi: 10.3389/fmolb.2019.00160

25. Pickup MW, Mouw JK, Weaver VM. The Extracellular Matrix Modulates the Hallmarks of Cancer. EMBO Rep (2014) 15:1243-53. doi: 10.15252/ embr.201439246

26. Forner A, Reig M, Bruix J. Hepatocellular Carcinoma. Lancet (2018) 391:1301-14. doi: 10.1016/S0140-6736(18)30010-2

27. Wallace MC, Friedman SL. Hepatic Fibrosis and the Microenvironment: Fertile Soil for Hepatocellular Carcinoma Development. Gene Expr (2014) 16:77-84. doi: 10.3727/105221614X13919976902057

28. Caruana I, Savoldo B, Hoyos V, Weber G, Liu H, Kim ES, et al. Heparanase Promotes Tumor Infiltration and Antitumor Activity of CAR-Redirected T Lymphocytes. Nat Med (2015) 21:524-9. doi: 10.1038/nm.3833

29. Kirtane AR, Sadhukha T, Kim H, Khanna V, Koniar B, Panyam J. Fibrinolytic Enzyme Cotherapy Improves Tumor Perfusion and Therapeutic Efficacy of Anticancer Nanomedicine. Cancer Res (2017) 77:1465-75. doi: 10.1158/00085472.CAN-16-1646
30. Provenzano PP, Cuevas C, Chang AE, Goel VK, Von Hoff DD, Hingorani SR. Enzymatic Targeting of the Stroma Ablates Physical Barriers to Treatment of Pancreatic Ductal Adenocarcinoma. Cancer Cell (2012) 21:418-29. doi: 10.1016/j.ccr.2012.01.007

31. Rahbari NN, Kedrin D, Incio J, Liu H, Ho WW, Nia HT, et al. Anti-VEGF Therapy Induces ECM Remodeling and Mechanical Barriers to Therapy in Colorectal Cancer Liver Metastases. Sci Transl Med (2016) 8:360ra135. doi: 10.1126/scitranslmed.aaf5219

32. Kudo M, Finn RS, Qin S, Han KH, Ikeda K, Piscaglia F, et al. Lenvatinib Versus Sorafenib in First-Line Treatment of Patients With Unresectable Hepatocellular Carcinoma: A Randomised Phase 3 non-Inferiority Trial. Lancet (2018) 391:1163-73. doi: 10.1016/S0140-6736(18)30207-1

33. Llovet JM, Ricci S, Mazzaferro V, Hilgard P, Gane E, Blanc JF, et al. Sorafenib in Advanced Hepatocellular Carcinoma. N Engl J Med (2008) 359:378-90. doi: 10.1056/NEJMoa0708857

Conflict of Interest: The authors declare that the research was conducted in the absence of any commercial or financial relationships that could be construed as a potential conflict of interest.

Publisher's Note: All claims expressed in this article are solely those of the authors and do not necessarily represent those of their affiliated organizations, or those of the publisher, the editors and the reviewers. Any product that may be evaluated in this article, or claim that may be made by its manufacturer, is not guaranteed or endorsed by the publisher.

Copyright (C) 2022 Yeow, Wu, Wang, Winteringham, Feindel, Tirnitz-Parker, Leedman, Ganss and Hamzah. This is an open-access article distributed under the terms of the Creative Commons Attribution License (CC BY). The use, distribution or reproduction in other forums is permitted, provided the original author(s) and the copyright owner(s) are credited and that the original publication in this journal is cited, in accordance with accepted academic practice. No use, distribution or reproduction is permitted which does not comply with these terms. 